\title{
Business as Usual \\ Der deutsche und französische Beitrag zur Korrumpierung des Oil for Food-Programms
}

\author{
JULIA ROLOFF ${ }^{* *}$
}

\section{The German and French Contribution to Corrupting the Oil for Food-Programme}

Between 1999 and 2002 more than 2300 companies from all over the world made illicit payments to the Iraqi government adding up to 1.8 billion US-Dollars. This was in spite of the fact that the UNSecurity Council had sanctioned the country with an embargo. The article discusses the contribution of German and French enterprises into corrupting the Oil for Food-Programme. For this purpose the transactions in the sales of Iraqi oil and in the purchase of humanitarian goods are analysed separately. Reflecting on the lessons learned in the Oil for Food-Programme, recommendations are given for the prevention of corruption in weak governance zones.

Keywords: Corruption, Transparency, Iraq, Oil for Food, United Nations, France

\section{Hilfe durch Handel: Das Oil for Food-Programm}

Zwischen 1996 und 2003 wurde das erste durch Handel finanzierte Hilfsprogramm in der Geschichte der Vereinten Nationen (VN) im Irak durchgeführt. Notwendig geworden war dieses Programm, weil die Vereinten Nationen ein Handelsembargo gegen den Irak verfügt hatten und eine Hungersnot im Irak ausbrach. Ziel des Embargos war es, den Irak dazu zu bewegen, mit Kuwait vertraglich Frieden zu schließen, nachdem Iraks Invasion in Kuwait durch eine internationale Koalition militärisch beendet worden war (vgl. Resolution 661 des VN-Sicherheitsrates). Eine Folge des Embargos war es, dass die Versorgung mit Nahrungsmitteln und anderen Gütern des täglichen Bedarfs im eigentlich wohlhabenden Irak weitgehend zusammenbrach. Eine Hungersnot trat auf, die die Regierung unter Präsident Saddam Hussein nicht verhindern konnte oder wollte.

Die Vereinten Nationen waren nun in eine Zwickmühle geraten. Gemäß der VNCharta bezweckt die Völkergemeinschaft, Frieden zu sichern sowie ökonomische,

Beitrag eingereicht am 02.06.2007; nach doppelt verdecktem Gutachterverfahren überarbeitete Fassung angenommen am 18.01.2008.

** Dr: Julia Roloff, ESC Rennes School of Business, 2, rue Robert d'Arbrissel, F-35065 Rennes, Tel.: +33-(0)29933-4830, Fax: +33-(0)29933-0824, E-Mail: julia.roloff@esc-rennes.fr, Forschungsschwerpunkte: Unternehmensethik, Corporate Social Responsibility, Stakeholdermanagement, Umwelt- und Sozialstandards, Korruption.

Für anregende und hilfreiche Kommentare bedanke ich mich bei Shirley van Buiren und zwei anonymen Gutachtern der zfwu. Die Arbeit an diesem Artikel wurde gefördert durch den ESC Rennes Management Research Fund. 
soziale, kulturelle und humanitäre Probleme zu lösen (vgl. Artikel 1 der Charta der Vereinten Nationen). Im Irak schienen die Vereinten Nationen an ihren eigenen Zielen zu scheitern. Das Oil for Food-Programm sollte dieses Dilemma lösen. ${ }^{1}$ Die Idee war, dass der Irak durch den kontrollierten Verkauf begrenzter Mengen Erdöls ein Hilfsprogramm finanziert. Angesichts der großen Erdölvorkommen im Lande, war es naheliegend nicht die internationale Gemeinschaft, sondern das Land selbst zur Kasse zu bitten. Der Irak verfügt über geschätzte 10 Prozent der weltweit bekannten Ölreserven und ist damit nach Saudi Arabien mit 22,9 Prozent und dem Iran mit 11,8 Prozent das Land mit den drittgrößten Erdölvorkommen weltweit (vgl. OPEC 2006). ${ }^{2}$

Gemäß den Richtlinien des Oil for Food-Programms durfte der Irak eine vom VNSicherheitsrat festgelegte Menge Erdöls zu einem ebenfalls festgelegen „fairen“ Preis auf dem Weltmarkt verkaufen (IIC-OFFP 2005a: 2). Die durch den Irak gewählten Käufer zahlten direkt auf ein Treuhandkonto, welches durch die Vereinten Nationen verwaltet wurde. Aus diesem Konto wurden nun humanitäre Güter bezahlt, die im Irak an Bedürftige verteilt wurden. ${ }^{3}$ Welche Güter eingeführt werden durften, entschied ebenfalls der Sicherheitsrat, um die Einführung zivil wie militärisch nutzbarer Güter zu verhindern bzw. zu kontrollieren. Im Rahmen des Oil for Food-Programms hatten gemäß der Resolution 986 alle Iraker ein Anrecht auf monatliche Lebensmittellieferungen. Gemäß einer Schätzung der Vereinten Nationen waren 60 Prozent der irakischen Haushalte von diesen Lieferungen komplett abhängig. ${ }^{4}$ Anfangs konzentrierte sich das Programm auf die Versorgung mit Lebensmitteln und Medikamenten sowie auf Maßnahmen in zentralen Bereichen wie z. B. Bildung, Landwirtschaft, Infrastruktur. Im Jahr 2002 wurden auch Anschaffungen in den Bereichen Kultur, Jugendarbeit, Rechtssystem usw. erlaubt, um eine weitere Verschlechterung der Lage zu

1 Eine Aufhebung der Sanktionen kam für den VN-Sicherheitsrat zu diesem Zeitpunkt nicht in Frage. Ein Schwiegersohn Saddam Husseins, General Hussein Kamal, war 1995 aus dem Irak geflohen und berichtete von einem umfangreichen irakischen Waffenprogramm (vgl. IIC-OFFP 2005b: 14).

2 Bis 2002 galt der Irak als das Land mit den weltweit zweitgrößten Erdölvorkommen. Die Entdeckungen neuer Vorkommen im Iran im Jahr 2002 und das Fehlen weiterer Explorationen im Irak nach 2001 führten zu dem Platzwechsel (vgl. OPEC 2006). Grundsätzlich müssen Angaben zu bekannten Ölreserven mit Vorsicht beurteilt werden, da diese nicht nur von Explorationsaktivitäten und Ölförderungstechnologien abhängig sind, sondern weil die Bekanntgabe neuer Vorkommen auch einem politischem Kalkül unterliegt (vgl. Richards/Waterbury 1991: 60).

3 Die Erlöse aus dem Verkauf von Erdöl wurden wie folgt verwendet: 72 Prozent für humanitäre Hilfe (59 Prozent für die 15 Provinzen, in denen irakische Behörden das Programm verwalteten, und 13 Prozent für die drei nördlichen Provinzen, wo die Vereinten Nationen die Verteilung und Verwaltung der Hilfsmittel übernahmen), 25 Prozent für Reparationszahlungen, 2,2 Prozent für administrative Kosten auf Seiten der Vereinten Nationen und 0,8 Prozent für Waffeninspektionen (vgl. Office of the Iraq Programme Oil for Food, www.un.org/Depts/oip/background/ index.html).

4 Diese Informationen stammen von der Internet-Seite des Office of the Iraq Programme Oil for Food: www.un.org/Depts/oip/sector-food.html. Das Programm konnte erfolgreich die Hungersnot eindämmen. Dieselbe Internet-Seite dokumentiert, dass die bereitgestellten Lebensmittel zum Beginn des Programms 1996 eine Versorgung mit $1200 \mathrm{kcal}$ pro Person und Tag sicherstellten. Bis 2002 erhöhte sich die Anzahl auf 2200 kcal, wobei die Lebensmittelpreise im Irak gleichzeitig sanken. 
verhindern. Nach dem Sturz Saddam Husseins durch eine durch die USA angeführte Koalition wurde das Oil for Food-Programm im November 2003 offiziell durch die Vereinten Nationen beendet und den irakischen Behörden übergeben.

Anfang 2004 tauchten Zeitungsberichte über eine Korrumpierung des Oil for FoodProgramms auf. Ein Nutznießer von unlauteren Geschäftspraktiken soll Kojo Annan, der Sohn des damals amtierenden VN-Generalsekretärs Kofi Annan, gewesen sein. ${ }^{5}$ Im April 2004 setzte Kofi Annan eine unabhängige Kommission ein, die den Fall untersuchen und Fakten sammeln sollte. Geleitet wurde das Komitee durch Paul A. Volcker - ein ehemaliger U. S. Federal Reserve Chairman. Weitere Mitglieder waren der Südafrikaner Richard Goldstone, der zuvor als Chefankläger der VNKriegstribunale in Ex-Jugoslawien und in Ruanda tätig gewesen war, und der Schweizer Professor Mark Pieth, der einen Lehrstuhl für Strafrecht und Kriminologie an der Universität Basel innehat. ${ }^{6}$ In September und Oktober 2005 veröffentliche das Komitee zwei umfangreiche Berichte und ausführliches Datenmaterial (insgesamt 2570 Seiten) über illegale Zahlungen an den Irak (vgl. IIC-OFFP 2005a) und die Gründe dafür, dass diese Zahlungen durch die VN-Verantwortlichen weder verhindert noch geahndet wurden (vgl. ICC-OFFP 2005b, 2005c, 2005d \& 2005e).

Im Folgenden wird auf Grundlage dieser Berichte dargestellt, wie es zu einer Korrumpierung des Oil for Food-Programms gekommen ist. Dabei wird exemplarisch die Rolle deutscher und französischer Unternehmen und Mittelsmänner beleuchtet. Insbesondere wird der Frage nachgegangen, in welchem Ausmaß die Unternehmen am Aufbau korrupter Strukturen beteiligt waren. Zuletzt wird beschrieben, wie Unternehmen, die in Konfliktzonen tätig sind, einen Beitrag zur Korruptionsprävention und -bekämpfung leisten können.

\section{Die Korrumpierung des Programms}

Die Untersuchungskommission konnte belegen, dass der Irak insgesamt 1,8 Milliarden US-Dollar aus dem Oil for Food-Programm in eigene Taschen geschleust hat. Nur ein kleiner Anteil - 229 Millionen US-Dollar - wurde mit Zuschlägen auf Erdölverkäufen erwirtschaftet (vgl. IIC-OFFP 2005a: 1). Das Gros der Einnahmen wurde beim Einkauf humanitärer Güter eingenommen, wobei die Schmiergelder als Gebühren getarnt wurden. Die irakischen Behörden verlangten zunächst für Güter, die auf dem Seeweg angeliefert wurden, „Inland Transportation Fees“ in Höhe von etwa 10 Prozent des Auftragswerts, was die tatsächlichen Transportkosten deutlich überschritt (IIC-OFFP 2005a: 249). Nachdem so etwa 0,53 Milliarden US-Dollar eingesammelt worden waren, wurden diese Gebühren durch „After-Sales-Service-Fees“ ersetzt, die sich ebenfalls auf 10 Prozent des Auftragswerts bezifferten und insgesamt 1,02 Milliarden USDollar einbrachten (IIC-OFFP 2005a: 1). Auch in diesem Fall entstanden dem Irak in Wirklichkeit keine vergleichbaren Kosten für Dienstleistungen, die nach der Lieferung

5 Die Untersuchungskommission konnte die Vorwürfe gegen Kojo Annan nicht bestätigen und äußerte ihrerseits Zweifel an der Seriosität des Hauptinformanten, auf dessen Aussagen die Vorwürfe beruhten (vgl. IIC-OFFP 2005b).

6 Informationen zur Kommission sowie ihre Berichte finden sich auf der Internet-Seite des Independent Inquiry Committee (IIC): www.iic-offp.org. 
eines Produktes notwendig werden können (vgl. IIC-OFFP 2005a: 257). Um zu erklären, wie es dem Irak trotz strikter Sanktionen gelingen konnte, derart große Summen aus einem Nothilfeprogramm zu veruntreuen, wird nachfogend beschrieben, wie deutsche und französische Unternehmen zu Komplizen des Betrugs wurden.

\subsection{Aufschläge im Ölgeschäft}

Der Irak versuchte von Anfang an, politisches Kapital aus dem Hilfsprogramm zu schlagen. So bevorzugte das Land grundsätzlich Handelspartner aus solchen Ländern, die den Sanktionen kritisch gegenüber standen und bei einer Abstimmung im Sicherheitsrat bereit sein könnten, den Irak zu unterstützen. Folglich wurden Mitglieder des Sicherheitsrates wie Frankreich, Russland und China begünstigt (vgl. IIC-OFFP 2005a: 9). Dies ging so weit, dass es nicht nur zu mehr Vertragsabschlüssen kam mit Unternehmen, die aus diesen Ländern stammen, der Irak vergab auch Erdölkontingente an Individuen, Organisationen und politische Parteien, die sich kritisch zu dem Embargo äußerten (vgl. IIC-OFFP 2005a: 16). Deutsche Ölunternehmen gingen im Geschäft mit dem Irak leer aus.

Nach den russischen waren französische Unternehmen die zweitwichtigsten Einkäufer von Erdöl mit Aufträgen im Wert von ca. 4,4 Milliarden US-Dollar.7 74 Prozent des nach Frankreich verkauften Erdöls wurde von Total International Limited und SOCAP International Limited aufgekauft. Es gab zudem eine Reihe von Unternehmen, die sich als französisch ausgaben, um irakisches Öl kaufen zu können. ${ }^{8}$ Dass Schweizer Unternehmen Vitol S. A. beispielsweise kaufte Erdöl als, Vitol France', obwohl ein Unternehmen mit diesem Namens nicht existiert (vgl. IIC-OFFP 2005a: 47). Eine Reihe französischer Einzelpersonen erhielt ebenfalls Erdölkontingente, die diese gewinnbringend weiterverkauften. Es handelte sich hierbei vorwiegend um Personen, die aufgrund bestehender oder vergangener Tätigkeiten bei den Vereinten Nationen, im französischen Parlament und diplomatischen Dienst gute Kontakte zu politischen Entscheidungsträgern hatten. ${ }^{9}$ Der Irak verfolgte eindeutig das Ziel, die politische Stimmung zu seinem Gunsten zu beeinflussen, um ein baldiges Ende des Embargos zu erreichen.

Im Herbst 2000 begann die irakische Regierung, Aufschläge auf jeden verkauften Barrel Öl zu verlangen. Die Aufschläge wurden von insgesamt 139 Unternehmen auf Konten in Jordanien und Libanon überwiesen oder an einen Vermittler gezahlt (vgl.

7 Russische Unternehmen kauften etwa ein Drittel aller Erdölexporte unter dem Oil for FoodProgramm im Wert von 19,3 Milliarden US-Dollar (vgl. IIC-OFFP 2005a: 22).

8 Im Oktober 1998 wurden die irakischen Behörden durch einen Franzosen auf diese unter britischen und amerikanischen Firmen weit verbreitete Praxis aufmerksam gemacht mit der Folge, dass sich der Irak anschließend besser über seine Geschäftspartner informierte (vgl. IIC-OFFP 2005a: 47 f.).

9 Es handelt sich um Jean-Bernard Mérimée (Special Advisor des VN-Generalsekretärs), Charles Pasqua und Bernard Guillet (ehemaliger französischer Innenminister und sein Berater), Claude Kaspereit (Geschäftsmann und Sohn des Parlamentariers Gabriel Kaspereit), Serge Boidevaix (Consultant und ehemaliger Diplomat) und Gillies Munier (Generalsekretär des Vereins französisch-irakischer Freundschaft) (vgl. IIC-OFFP 2005a: 49-78). 
IIC-OFFP 2005a: 19). ${ }^{10}$ Hierunter waren auch französische Unternehmen. Staatsanwaltschaften in Italien, der Schweiz und den USA haben beispielsweise Beweise dafür gefunden, dass das französische Erdölunternehmen Total S. A. bis zu 1 Millionen USDollar über Dritte an irakische Behörden gezahlt hat. Als Vermittler fungierte unter anderem das italienische Unternehmen Betoil. Betoil soll allein mehr als 4,5 Millionen US-Dollar an illegalen Aufschlägen an den Irak gezahlt haben für Aufträge, die es für eine ganze Reihe internationaler Ölfirmen getätigt hat (vgl. Gatti 2007). Dank derartiger Täuschungsmanöver erhielten die irakischen Behörden das Geld, ohne dass die Vereinten Nationen davon erfahren mussten. ${ }^{11}$

Anfangs verlangten die Mitarbeiter der irakischen State Oil Marketing Organization (SOMO) Aufschläge in Höhe von 10 amerikanischen Cent pro Barrel. ${ }^{12}$ Im Dezember 2000 versuchte die SOMO 50 Cent pro Barrel zu erhalten und verursachte damit eine Krise (vgl. IIC-OFFP 2005a: 18). Alle namhaften Ölfirmen hörten auf, irakisches Erdöl zu kaufen. ${ }^{13}$ Selbst als die Zuschläge wieder gesenkt wurden, kamen viele frühere Einkäufer nicht wieder zurück. Stattdessen kauften vier Firmen, Bayoil Supply \& Training Limited aus den USA, die in Europa und der Karibik ansässige Taurus Group und die beiden Schweizer Unternehmen Glencore International AG und Vitol Group, den Großteil des irakischen Erdöls über Briefkastenunternehmen in Lichtenstein, Italien, Malaysia, der Schweiz u. a. auf (vgl. IIC-OFFP 2005a: 115-170). Ende 2002 flog der Betrug auf, nachdem der Irak 228,8 Millionen US-Dollar an Zuschlägen auf Erdölverkäufe im Wert von 64,2 Milliarden US-Dollar zur Seite geschafft hatte (vgl. IIC-OFFP 2005a: 21).

Die Untersuchungskommission hat die beteiligten Firmenvertreter und Begünstigte mit ihren Erkenntnissen konfrontiert und um eine Stellungnahme gebeten. Wie bei Korruptionsfällen üblich, sagten viele der Betroffenen, dass sie keine Zahlungen getätigt hätten (12,9 Prozent) oder geglaubt hatten, dies wären legale Ausgaben (1,4 Prozent). Die Mehrheit (72,7 Prozent) beantwortete die Anfrage gar nicht (vgl. IIC-OFFP 2005f: 3). Berichte Dritter legen allerdings nahe, dass damals unter Erdöleinkäufern offen über die Forderungen des Iraks gesprochen wurde und dass es bekannt war,

10 Im Verlauf des gesamten Programms kauften 248 Unternehmen irakisches Erdöl im Wert von insgesamt 64,2 Milliarden US-Dollar (vgl. IIC-OFFP 2005a: 1).

11 Die Untersuchungskommission stellt in ihrem Bericht detailliert dar, ab wann welche Informationen und Gerüchte die Verantwortlichen in den Vereinten Nationen erreicht haben und wie diese - oftmals unzulänglich - reagiert haben (vgl. IIC-OFFP 2005d).

12 Saddam Hussein setzte ein Komitee ein, welches die Höhe der Aufschläge beschloss. Dem Komitee gehörten der irakische Vizepräsident Taha Yassin Ramadan, die beiden Minister für Öl Tariq Aziz und Amer Rashid sowie Hikmat Al-Azzawi (Finanzminister), Mohammed Mehdi Daleh (Handelsminister) und Abd Al-Tawab Abdullah Al-Mullah Al-Hwaish (Minister für die Militärindustrialisierung) an (vgl. IIC-OFFP 2005a: 18).

$13 \quad$ Zu diesem Zeitpunkt fürchteten einige Mitarbeiter des Erdölministeriums und SOMOs um das Leben des verantwortlichen Ministers, sollte er nicht in der Lage sein, die Zahlungen einzutreiben (vgl. IIC-OFFP 2005a: 19). Die Untersuchungskommission berichtet wiederholt von Drohungen der Iraker gegen Handelspartner und Angehörige der Vereinten Nationen (vgl. IIC-OFFP 2005d: 52 f.). Angesichtes der zahlreichen Menschenrechtsverletzungen im Irak mussten diese Drohungen ernst genommen werden (vgl. Human Rights Watch 2000). 
dass die meisten Unternehmen zahlten, um zukünftige Geschäfte nicht zu gefährden (vgl. IIC-OFFP 2005a: 66). Ein fehlendes Problembewusstsein scheint die Schmiergeldzahlungen begünstigt zu haben. Beispielsweise wird der Generalsekretär des Vereins französisch-irakischer Freundschaft zitiert mit einem Statement, er habe von illegalen Aufschlägen im Ölgeschäft gehört, aber selbst wenn diese für seine Ölkontingente gezahlt worden seien, sei dies nicht sein Problem (vgl. IIC-OFFP 2005a: 74).

\subsection{Schmiergeldzahlungen im Handel mit humanitären Gütern}

Die Korrumpierung des Oil for Food-Programms begann Mitte 1999, als irakische Behörden beim Einkauf von humanitären Gütern erstmals Inland Transportation Fees (ITF) in Höhe von mindestens 10 Prozent des Auftragswerts von den Lieferanten forderten. ${ }^{14}$ Auf diese Weise konnte deutlich mehr Geld eingenommen werden, als der Transport der Güter vom Hafen Umm Qasr zu ihren Einsatzorten kostete (vgl. IIC-OFFP 2005a: 249). Ein Jahr später wurde das Programm auf Geheiß des irakischen Vizepräsidenten Ramadan auch auf Güter, die nicht in Umm Qasr angeliefert wurden, ausgeweitet (vgl. IIC-OFFP 2005a: 276). Die Forderungen wurden nun AfterSales-Service-Fees (ASSF) genannt und betrugen ebenfalls in der Regel 10 Prozent des Auftragswerts. Eine Zeit lang forderten die Behörden beide Gebühren von den Unternehmen und erhielten so stattliche 20 Prozent. Die ITF wurden aber im Laufe der Zeit eingestellt. Ein Blick in die Tabelle 8 des Berichts der Untersuchungskommission (vgl. IIC-OFFP 2005a) zeigt, dass die eingeforderten und gezahlten Beträge variieren. Gelegentlich haben Unternehmen sogar mehr als 20 Prozent des Auftragswerts als Schmiergeld bezahlt. Andere Aufträge wurden abgewickelt, ohne dass die angeblichen Gebühren vollständig abgegolten wurden.

Bei den Geschäften mit humanitären Gütern war es komplizierter als im Ölhandel, die illegalen Zahlungen vor den Vereinten Nationen zu verheimlichen, da die Waren direkt vom Treuhandkonto bezahlt wurden. Die Geschäfte zwischen dem Irak und den internationalen Zulieferern liefen daher wie folgt ab. Nachdem die irakischen Behörden einen Zulieferer für ein bestimmtes Gut ausgewählt hatten, traten sie mit ihm in Kontakt und erklärten, dass sie auf diesen Auftrag Gebühren erheben, die direkt an den Irak zu zahlen sind. Die Höhe dieser Zahlungen wurde in vielen Fällen in einer Nebenabsprache schriftlich fixiert. ${ }^{15}$ Die Zulieferer wurden dazu angehalten, den Auftragswert in ihrer Rechnung an die Vereinten Nationen um den Betrag der Gebühren zu erhöhen. Allerdings wurde diese Erhöhung in der Regel nicht kenntlich gemacht, indem ein Posten für ASSF oder ITF aufgeführt wird. Im Gegenteil: Die im Bericht abgedruckten Belege - beispielsweise von Siemens France - geben nur einen Gesamtrechnungsbetrag an, der im genannten Fall den Warenwert um 10 Prozent übersteigt

14 Die Untersuchungskommission macht in ihrem Bereicht nur bei wenigen Unternehmen konkrete Angaben zur Höhe der gezahlten Inland Transportation Fees (ITF), da die Datenlage dies in vielen Fällen nicht zulässt. Auch im Fall der After-Sales-Service-Fees (ASSF) ist nur ein Teil der Zahlungen belegt und die übrigen wurden aufgrund der z.B. aus Nebenabsprachen vorliegenden Informationen geschätzt (vgl. IIC-OFFP 2005a: 92f.).

15 Die Untersuchungskommission hat fast 1000 Nebenabsprachen für ihren Bericht ausgewertet (vgl. IIC-OFFP 2005a: 290). 
(vgl. IIC-OFFP 2005a: 376). ${ }^{16}$ Die Vereinten Nationen überwiesen daraufhin den Rechnungsbetrag an das Unternehmen. Das Unternehmen zahlte anschließend die Gebühren an irakisch kontrollierte Konten in Jordanien oder den Libanon oder gab das Geld einem Mittelsmann (vgl. IIC-OFFP 2005a: Tabelle 8).

Wie schon beim Verkauf des Erdöls liess sich der Irak auch bei der Auftragsvergabe an Zulieferer humanitärer Güter von politischen Überzeugungen leiten. Aus diesem Grund wurden beispielsweise französische Zulieferer den deutschen vorgezogen. Dies spiegelt sich in der Anzahl deutscher und französischer Unternehmen wider, die in den Skandal verwickelt sind. Weltweit haben 2253 Zulieferer humanitärer Güter die angeblichen Gebühren gezahlt (vgl. IIC-OFFP 2005a: 1). ${ }^{17}$ Abbildung 1 fasst auf Grundlage des Abschlussberichts der Untersuchungskommission die Informationen zu den deutschen und französischen Unternehmen zusammen. Es handelt sich nur um Unternehmen, die illegale Zahlungen an den Irak geleistet haben. Handelpartner vor 1999 und nach 2002 werden hier nicht berücksichtigt.

\begin{tabular}{|c|c|c|}
\hline & Deutsche Unternehmen & Französische Unternehmen \\
\hline $\begin{array}{c}\text { Anzabl } \\
\text { involvierter Unternebmen }\end{array}$ & $\begin{array}{l}59 \text { Unternehmen und } \\
5 \text { Tochterunternehmen } \\
\text { mit Sitz im Ausland }\end{array}$ & $\begin{array}{l}167 \text { Unternehmen mit Sitz } \\
\text { in Frankreich und } 10 \text { mit Sitz } \\
\text { in weiteren Ländern }\end{array}$ \\
\hline $\begin{array}{l}\text { Auftragswert insgesamt } \\
\text { (ohne Zuschläge) }\end{array}$ & 193944330 US-Dollar & 787905530 US-Dollar \\
\hline $\begin{array}{c}\text { Gezablte ASSF } \\
\text { (in \% des Auftragswertes) }\end{array}$ & $\begin{array}{c}11977570 \text { US-Dollar } \\
(6,2 \%) \\
\end{array}$ & $\begin{array}{c}48367527 \text { US-Dollar } \\
(6,1 \%) \\
\end{array}$ \\
\hline $\begin{array}{l}\text { Anzabl der Unternebmen, } \\
\text { die ITF gezablt haben }\end{array}$ & $\begin{array}{l}18 \text { Unternehmen } \\
(26,9 \%)\end{array}$ & $\begin{array}{c}67 \text { Unternehmen } \\
(37,9 \%)\end{array}$ \\
\hline
\end{tabular}

Abbildung 1: Illegale Zablungen im Handel mit bumanitären Gütern'18

Grundsätzlich können anhand der vorliegenden Daten keine wesentlichen Unterschiede zwischen dem Verhalten deutscher und französischer Manager ausgemacht werden. In beiden Ländern entschieden Führungskräfte, den Forderungen der Iraker

16 An andere Stelle im Bericht wird angegeben, dass die Gebühren in manchen Rechnungen als solche ausgewiesen waren und von den Vereinten Nationen erstattet wurden (vgl. IIC-OFFP 2005a: 250).

17 Insgesamt haben im Rahmen des Oil for Food-Programms weltweit 3614 Unternehmen Güter im Wert von 34,5 Milliarden US-Dollar an den Irak geliefert. Dies Zahl schließt Geschäfte vor 1999 mit ein (vgl. IIC-OFFP 2005a: 1).

18 Die Daten stammen aus IIC-OFFP 2005a: Tabelle 8. Die Angaben zu deutschen Tochterunternehmen im Ausland wurden auf Grundlage zusätzlicher Internetrecherchen gemacht; eine Garantie auf Vollständigkeit kann nicht gegeben werden. In Deutschland ansässige Tochterunternehmen wurden nicht mitgezählt. Angaben zum Auftragswert sowie zu gezahlten ASSF und ITF beziehen sich auf Mutter- und Tochterunternehmen. Die Angaben zu den französischen Unternehmen wurden ohne weitere Korrekturen aus Tabelle 8 übernommen. 
nachzugeben. Die Zahlungsmoral war in beiden Ländern gut. Allein die ASSF brachten gut sechs Prozent des Auftragswertes ein. Die genaue Höhe der gezahlten ITF konnte nicht ermittelt werden, aber gut ein Viertel der deutschen und fast 38 Prozent der französischen Firmen sollen diese laut Untersuchungsbericht bezahlt haben.

Die Unternehmen reagierten unterschiedlich, als die Untersuchungskommission sie mit ihren Ergebnissen konfrontierte. Ein Teil gab an, von den Zahlungen nichts gewusst zu haben, da diese durch Vermittler oder Mitarbeiter veranlasst worden seien. So erklärte beispielsweise der Vorsitzende von DaimlerChrysler AG von einer durch einen Mitarbeiter unterzeichneten Nebenabsprache nichts zu wissen. Der betreffende Mitarbeiter „expressed his confusion about the programm“ (IIC-OFFP 2005a: 371) gegenüber den Ermittlern und gab an, sich an eine solche Vereinbarung nicht erinnern zu können. Als ihm zwei Nebenabsprachen vorgelegt wurden, bestätigte er, dass die Unterschriften unter ihnen wie die Seine aussehen, zweifelte aber an ihrer Authentizität mit dem Hinweis „you can do funny things with a computer“ (IIC-OFFP 2005a: 370). Laut Bericht der Untersuchungskommission unterzeichnete dieser Mitarbeiter insgesamt drei Nebenabsprachen bezüglich Zahlungen über mehr als $80000 €$. Die DaimlerChrysler AG hielt sich allerdings nur an eine dieser Nebenabsprachen und zahlte etwa $6950 €$ an den Irak (vgl. IIC-OFFP 2005a: 371). Eine Erklärung hierfür könnte sein, dass andere Mitarbeiter im Unternehmen oder ein Managementsystem zur Korruptionsprävention die Unregelmäßigkeit als solche erkannt und weitere Zahlungen verhindert haben. DaimlerChrysler machte hierzu keine weiteren Angaben.

Viele Unternehmen arbeiteten mit Vermittlern (,agents“, IIC-OFFP 2005a: 250), welche die Aufgabe hatten, die Geschäfte mit dem Irak abzuwickeln. Es ist mit Einschränkungen plausibel, dass die Unternehmen von Zahlungen, die durch Vermittler veranlasst wurden, keine Kenntnis haben mussten. Andere Zulieferer humanitärer Güter gaben an, geglaubt zu haben, dass es sich bei ITF und ASSF um legitime Gebühren gehandelt habe und dass diese im Rahmen des Oil for Food-Programms erlaubt gewesen seien (vgl. IIC-OFFP 2005a: 250). Ein Teil der Unternehmen leugnete, derartige Zahlungen veranlasst zu haben oder zweifelte an der Echtheit der Beweise. Andere gaben zu, Schmiergelder bezahlt zu haben. Diese seien „the cost of doing business with Iraq and (...) all companies had to pay these fees in order to obtain goods contracts from Iraq" (IIC-OFFP 2005a: 251).

\section{Die Suche nach den Schuldigen}

Korruption wird zumeist als ein Strukturproblem diskutiert, dessen Bekämpfung das Zusammenspiel zahlreicher Akteure voraussetzt (vgl. Klitgaard 1997; Theobald 2002; Aidt 2003). Ist ein Markt von korrupten Strukturen geprägt, ist es Anbietern nahezu unmöglich im Wettbewerb zu bestehen, ohne selbst Schmiergelder zu zahlen. Als mögliche Ursachen für Korruption werden ineffiziente Bürokratien, unterbezahlte Beamte, fehlende Kontroll- und Sanktionssysteme, ein Mangel an Transparenz und effizienter Berichtlegung sowie das Fehlen einer starken und kritischen Zivilgesellschaft und unabhängiger Medien genannt (vgl. Theobald 2002; Eigen 2002). Wie korrupte Strukturen sich konkret entwickeln, wird in der Literatur mangels geeigneter Beispiele nicht erklärt. Der vorliegende Fall des Oil for Food-Programms schließt diese Erkenntnislücke. 
Der Untersuchungsbericht der von Volcker geleiteten Kommission beschreibt im Detail mit welcher Geschwindigkeit das Zahlen von Schmiergeldern durch irakische Behörden eingeführt werden konnte und wie gering der Widerstand seitens der Unternehmen gewesen war. Es darf nicht vergessen werden, dass zwischen 1996 und 1999 das Programm ohne nennenswerte Korruptionsvorfälle verlief. Dies war der Fall, obwohl der Irak als ein korruptes Land gilt. Im von Transparency International durchgeführten Landesvergleich, dem Corruption Perceptions Index, lag der Irak in den letzten Jahren wiederholt auf einem der letzten Plätze (vgl. www.transparency.org). Ähnlich wie es möglich ist, dass einzelne Märkte - beispielsweise die Baubranche in Deutschland - in ansonsten transparenten Ländern von Korruption verstärkt betroffen sind, scheint es im Irak möglich zu sein, in einem geschützten Raum wie dem stark regulierten Oil for Food-Programm, Geschäfte ohne Schmiergeldzahlungen abschließen zu können. Die transparente Abwicklung war sowohl für die Lieferanten als auch für die Nutznießer des Programms vorteilhaft, da auf diese Weise die gesamten Einnahmen aus dem Erdölverkauf für den Einkauf humanitärer Güter zur Verfügung standen. Wie kam es nun dazu, dass weder die Vereinten Nationen noch die involvierten Unternehmen, den Korrumpierungsversuch der irakischen Behörden vereitelt haben?

\subsection{Whistleblower wurden nicht gehört}

Mit der Veröffentlichung des Berichts der Untersuchungskommission im Oktober 2005 begann die öffentliche und rechtliche Aufarbeitung des Skandals. Obwohl die Kommission deutlich feststellt, dass die Korrumpierung des Programms durch Saddam Husseins Regierung initiiert wurde, deckt sie eine Mitschuld weiterer Akteure auf. So wird deutlich, dass die Vereinten Nationen das Thema Korruption im Irak vernachlässigt hatten. Besondere Schuld trifft in diesem Zusammenhang die Verantwortlichen für die Verwaltung des Oil for Food-Programms. Sie weigerten sich lange, den Hinweisen und Gerüchten zu Schmiergeldzahlungen nachzugehen, obwohl sogar ihre Mitarbeiter sie dazu aufforderten (vgl. IIC-OFFP 2005d: 79 ff. \& 93 ff.). Es war die Ansicht des Excecutive Director des Programms Benon Sevan, dass „his mandate was to get food and medication to the Iraqi people and it was not his job to report about the kickbacks, which he saw as part of the Iraqi culture" (IIC-OFFP 2005d: 78). Rückblickend muss anerkannt werde, dass er den Auftrag, die irakische Bevölkerung zu versorgen, erfüllt hat. In der öffentlichen Wahrnehmung jedoch wird das Oil for Food-Programm in erster Linie als Korruptionsskandal in Erinnerung bleiben, eben weil er nicht rechtzeitig eingegriffen hat.

Nun ist Korruptionsbekämpfung nicht nur eine Aufgabe der Vereinten Nationen. Spätestens seit der Verabschiedung der OECD-Konvention zur Bekämpfung der Bestechung ausländischer Amtsträger (vgl. OECD 1998) und der daraus resultierenden Gesetzesänderungen in den OECD-Mitgliedstaaten sollten international tätige Unternehmen Maßnahmen zur internen Korruptionsprävention ergriffen haben. Trotzdem haben zahlreiche mittelständische und multinationale Unternehmen Gelder an den Irak überwiesen und zu diesem Zweck ihre Rechnungen an die Vereinten Nationen manipuliert. Ein Beispiel ist die Siemens AG, welche bereits damals über ein umfangreiches Risikomanagementsystem zur „Prävention von Vermögensveruntreu- 
ung und Missbrauch“ verfügte (Siemens 2006: 142). Das Unternehmen hat - ihre Töchter im Ausland eingerechnet - insgesamt 609558 US-Dollar an den Irak gezahlt (vgl. IIC-OFFP 2005a: Tabelle 8). Siemens stellt in seinem Geschäftsbereicht 2006 fest, dass sein Managementsystem versagt hat und verspricht dieses in $2007 \mathrm{zu}$ verbessern (vgl. Siemens 2006: 142).

Besonders bedenklich ist, dass sich nur wenige Unternehmen die Mühe machten, die Vereinten Nationen über die Bestechungsversuche der Iraker zu informieren. Eine Ausnahme war das schwedische Unternehmen Scania CV AB, welches im Sommer 2000 - kurz nach der Einführung der ASSF - das fürs Oil for Food-Programm verantwortliche Büro bei den Vereinten Nationen kontaktierte. ${ }^{19}$ Scania berichtete, dass irakische Behörden Gebühren in Höhe von 15 Prozent des Auftragswerts von dem Unternehmen verlangt hatten und dass es die Zahlung verweigert habe (vgl. IICOFFP 2005d: 77). Im Laufe des Jahres unterrichteten etwa sechs weitere Unternehmen die Vereinten Nationen über Geldforderungen der Iraker. Angesichts der fast 2400 Unternehmen, die irreguläre Zahlungen an den Irak geleistet haben, ist diese Zahl ernüchternd.

Einige der Geschäftsleute haben möglicherweise aus Angst geschwiegen. So berichtete ein Hinweisgeber, dass er, nachdem ihm die illegale Nebenabsprache vorgelegt wurde, zu einem hochrangigen irakischen Amtsträger gebracht wurde. Dieser machte deutlich, dass das Unternehmen die geforderte Summe zahlen musste, um den Auftrag zu erhalten. Sollte er sich weigern, die Nebenabsprache zu unterschreiben, oder sich entscheiden, sie bekannt zu machen, würde sein Unternehmen keine weiteren Aufträge mehr erhalten (vgl. IIC-OFFP 2005d: 79). Dafür, dass derartige Drohungen regelmäBig ausgesprochen wurden, gibt es allerdings keine Hinweise.

\subsection{Geschäfte um jeden Preis?}

Trotz zahlreicher Unschuldsbehauptungen und Ausreden ist die Behauptung der Unternehmen, von dem illegalen Charakter der Zahlungen nichts gewusst zu haben, wenig überzeugend. Auch scheint es nicht plausibel zu sein, dass sich die Vermittlung von Verträgen durch Agenten um mehr als zehn Prozent des Auftragswertes verteuern konnte, ohne dass dies zu Fragen führte. Im Gegenteil: Es ist üblich, Vermittler einzuschalten, um Schmiergeldzahlungen zu verschleiern. Typischerweise wird dabei den Mittelsmännern vertraglich untersagt, Schmiergelder zu zahlen, obwohl dies deren wesentliche Dienstleistung ist. Derartige Unterlassungserklärungen mussten auch die Vermittler im Erdölgeschäft unterzeichnen (vgl. IIC-OFFP 2005a: 4). Vor diesem Hintergrund behaupteten einige der Einkäufer der Untersuchungskommission gegenüber, sie hätten die Erhöhung des Erdölpreises für einen Markteffekt gehalten. Ein Mitarbeiter von Bayoil hingegen gab offen zu, dass der „Disclaimer“ erst nachträglich

19 Bereits früher hatte es nach der Einführung von ITF einen entsprechenden Hinweis von Seiten des Canadian Wheat Boards gegeben (vgl. IIC-OFFP 2005a: 73f.). Auch österreichische Diplomaten kontaktierten das Büro des Oil for Food-Programms im Januar 2000, um zu erfragen, ob die Zahlungen des österreichischen Unternehmens Marquette Hellige Ges.ö.b.H. in Höhe von 15 Prozent des Auftragswerts erlaubt gewesen seien (vgl. IIC-OFFP 2005a: 75). 
in die Verträge aufgenommen wurde, um die Schmiergeldzahlungen zu verheimlichen (vgl. IIC-OFFP 2005a: 4).

Es zeigte sich, dass die Erdölunternehmen nicht bereit waren, jeden Preis zu zahlen. Aufschläge in Höhe von 50 amerikanischen Cent pro Barrel waren zuviel. Vermutlich war dieser Preis in Verbindung mit einer möglichen Rufschädigung zu hoch. Trotzdem konnten die vier Abnehmer irakischen Erdöls ihr Produkt weiterverkaufen, was zeigt, dass auch beim Ölpreis ein Verhandlungsspielraum besteht. Der größte Teil der vom Irak eingenommenen Gelder stammte jedoch nicht aus den Taschen der Unternehmen, sondern aus den durch die Vereinten Nationen verwalteten Erlösen aus den Ölverkäufen. Dies erklärt, warum im Handel mit humanitären Gütern Aufschläge über 10 Prozent des Auftragwerts - gelegentlich auch über 20 Prozent und mehr akzeptiert wurden. Dass hierfür die Rechnungen umgeschrieben werden mussten, schien in den Augen der Handelspartner nicht problematisch zu sein.

Diese Naivität bzw. Dreistigkeit hat ihren Preis. Anlässlich des Berichts der Untersuchungskommission untersuchen Staatsanwaltschaften weltweit die Vorgänge. In mehreren Ländern kam es bereits zu Vergleichen mit Unternehmen, die so einen Prozess verhindern konnten. So zahlte das amerikanische Gasunternehmen El Paso 7,7 Millionen US-Dollar Buße, weil es etwa 4,3 Millionen Euro Aufschläge für seine Erdölkontingente an den Irak gezahlt hatte (vgl. Masters 2007). In Deutschland sollen sechs Unternehmen Vergleiche geschlossen haben (vgl. Döbler 2007). Über die Höhe der gezahlten Bußen herrscht Unklarheit. Der Tagesspiegel beziffert sie auf insgesamt 750000 Euro (vgl. Döbler 2007); die Süddeutsche Zeitung berichtet von zwei Strafverfahren der Staatsanwaltschaft Bochum die mit Zahlungen von etwa 200000 Euro und 400000 Euro abgeschlossen worden seien. Viele Firmen gehen trotz aller Bemühungen straffrei aus. In Deutschland wurden insgesamt 45 Fälle staatsanwaltschaftlich untersucht und viele Verfahren wurden inzwischen eingestellt (vgl. Leyendecker/Krüger 2007).

Auch in Frankreich wurden die Staatsanwälte aktiv. Im Oktober 2006 wurde der Vorstandsvorsitzende der Total SA Christophe de Margerie für zwei Tage in Untersuchungshaft genommen und bezüglich der Zahlungen an den Irak befragt (vgl. Gatti 2007). Nach diesen ersten Ermittlungen, in deren Rahmen auch der ehemalige Leiter von SOMO Tarek Aziz befragt wurde, leitete die Staatsanwaltschaft Strafverfahren gegen insgesamt 14 Total-Mitarbeiter ein (vgl. Libération 2007). De Margerie und den anderen Total-Mitarbeitern wird Komplizenschaft bei der Unterschlagung von Unternehmensvermögen sowie Komplizenschaft bei der Bestechung ausländischer Amtsträger vorgeworfen (vgl. Gatti 2007).

Deutsche Staatsanwaltschaften nahmen in mehreren Fällen Abstand vom Vorwurf der Korruption. Sie untersuchten die Vorfälle vorwiegend als Verstöße gegen Paragraph 34 des Außenwirtschaftsgesetztes. In Abschnitt vier werden Freiheitsstrafen von sechs Monaten bis zu fünf Jahren angedroht, für Zuwiderhandlungen gegen ,einer vom Sicherheitsrat der Vereinten Nationen nach Kapitel VII der Charta der Vereinten Nationen (...) beschlossenen wirtschaftlichen Sanktionsmaßnahme“ ( $\$ 34$ AWG). Der Grund für diese Entscheidung war vermutlich, dass die Definition von Bestechung, die aus den Paragraphen 299 und 334 des Strafgesetzbuches hervorgeht, nicht auf die Vorgänge im Oil for Food-Skandal zutrifft. Bestechung liegt demnach vor, wenn je- 
mand „einem Amtsträger (...) einen Vorteil für diesen oder einen Dritten als Gegenleistung dafür anbietet, verspricht oder gewährt, (dafür dass) er eine Diensthandlung vorgenommen hat oder künftig vornehme und dadurch seine Dienstpflichten verletzt hat oder verletzen würde“ (\$334 StGB). Da es im Falle der irakischen Beamten zu keiner Verletzung der Dienstpflicht kam, sondern diese vielmehr von höchster Stelle beauftragt waren, Gelder einzutreiben, liegt im juristischen Sinne keine Bestechung vor.

Der französische Gesetzestext lässt einen größeren Interpretationsspielraum. Nach Artikel 435-3 des Code Penal kann wegen Korruption belangt werden, wer ohne Anrecht im Rahmen internationaler Geschäftsbeziehungen Angebote, Versprechungen oder Geschenke einer Amtsperson direkt oder indirekt anbietet, damit diese eine Amtshandlung ausführt bzw. unterlässt oder um Aufträge in einem Markt zu bekommen bzw. zu behalten oder um sich einen anderen Vorteil im internationalen Handel zu sichern. ${ }^{20}$ Der vorliegende Tatbestand kann daher nach französischem Recht als Korruption verfolgt werden.

\section{Regeln für Geschäfte in Konfliktzonen}

In vielerlei Hinsicht handelt es sich bei der Korrumpierung des Oil for FoodProgramms um einen einzigartigen Fall. Es war das erste Hilfsprogramm in einem mit einem Handelsembargo belegten Land, das durch kontrollierten Handel finanziert wurde. Sowohl die Vereinten Nationen als auch die involvierten Unternehmen haben hier neue Erfahrungen gesammelt. Die Annahme der Beteiligten, dass man Korruption zu einem gewissen Grade ignorieren bzw. akzeptieren durfte, wurde widerlegt. Die Aufdeckung der Korruption kam für die Beteiligten überraschend. Auslöser waren Gerüchte und Medienberichte, die behaupteten, dass Kofi Annan durch dessen Sohn in eine Korruptionsaffäre verwickelt sei. Es ist naheliegend, dass es das Ziel dieser Gerüchte war, das Ansehen des Nobelpreisträgers zu schädigen, der mit den USA zu diesem Zeitpunkt nicht zuletzt wegen ihrer Irakpolitik streng ins Gericht ging. Die Reaktion des Generalsekretärs, die Vorwürfe lückenlos aufklären zu lassen, führte zur Aufdeckung von Geschäftspraktiken, die für sich genommen, möglicherweise nicht Anlass einer derartigen Untersuchung gewesen wären. ${ }^{21}$

Der Gesetzestext lautet im Original: „Pour l'application de la convention sur la lutte contre la corruption d'agents publics étrangers dans les transactions commerciales internationales signée à Paris le 17 décembre 1997, est puni de dix ans d'emprisonnement et de 150000 euros d'amende le fait de proposer sans droit, à tout moment, directement ou indirectement, des offres, des promesses, des dons, des présents ou des avantages quelconques pour obtenir d'une personne dépositaire de l'autorité publique, chargée d'une mission de service public, ou investie d'un mandat électif public dans un Etat étranger ou au sein d'une organisation internationale publique, qu'elle accomplisse ou s'abstienne d'accomplir un acte de sa fonction, de sa mission ou de son mandat ou facilité par sa fonction, sa mission ou son mandat, en vue d'obtenir ou conserver un marché ou un autre avantage indu dans le commerce international. (...)“ (Artikel 435-3 Code Penal).

Insidern zufolge sind Schmiergeldzahlungen im internationalen Handel in vielen Ländern und Industrien üblich. Oftmals werden hierfür Vermittler eingesetzt, damit das Unternehmen zumindest in der Papierform an der Korruption unbeteiligt ist. Diese Praktiken sind derart verbreitet, dass der Eindruck entstehet, dass sie auch von öffentlicher Seite akzeptiert werden, solange keine konkreten Zahlungen öffentlich werden (Quelle: persönliche Gespräche). 
Die durch die Untersuchungskommission veröffentlichten Daten lassen die Vermutung zu, dass es sich hier nur um die Spitze des Eisbergs handelt. Wenn Unternehmen bereit sind, innerhalb eines streng geregelten Hilfsprogramms Gelder zu hinterziehen und einer Regierung zukommen zu lassen, der Folter, Massentötungen und offensive Kriegsführung vorgeworfen werden, wie verhalten sie sich dann in anderen Ländern, mit schwachen Regierungen und korrupten Strukturen? Vermutlich genauso wie im Irak: Sie zahlen, was von ihnen gefordert wird, und versuchen, diese Zahlungen mittels Agenten und schwarzen Konten zu vertuschen. Dieses Problem ist allgemein bekannt und scheint trotzdem unlösbar zu sein. Unternehmen und Regierungen sehen sich in einem Gefangenendilemma, in dem die erste Person, die sich an eine im Sinne des Gemeinwohls wünschenswerte Verhaltensnorm hält, sich deutliche Nachteile einhandelt, solange auch nur ein Teil der anderen Akteure, sich weiterhin Gemeinwohl schädigend verhält (vgl. Homann/Suchanek 2005: 33). Einige Vorschläge zur Lösung des Problems gibt es trotzdem und ein Instrument steht dabei im Mittelpunkt: Transparenz.

Sowohl von Seiten der OECD (vgl. OECD 2006 \& 2000) als auch von Seiten der NGO-Koalition Publish What You Pay (vgl. Global Witness 2005) wird darauf gedrungen, Zahlungen an Vermittler und Behörden offenzulegen. ${ }^{22}$ Die Idee ist, dass durch die Offenlegung Zahlungen verschiedener Unternehmen vergleichbar werden und auf diese Weise Schmiergeldzahlungen offensichtlich werden. Die Voraussetzung hierfür ist, dass Kenntnis darüber besteht, unter welchen Voraussetzungen Zahlungen in einer bestimmten Höhe und an bestimmte Adressaten als normal anzusehen sind. Solange diese Erkenntnisse fehlen, erscheinen die Zahlungsströme eines vollständig korrupten Marktes genauso normal wie die eines von Schmiergeld freien Marktes.

Aber auch ohne entsprechender Gesetzgebung oder kollektiven Vereinbarungen zur Offenlegung von Zahlungsströmen können Unternehmen aktiv werden. Es ist möglich, durch einen Führungsstil der Redlichkeit, eine Atmosphäre im Unternehmen zu schaffen, in der Ethikkodizes und die dazugehörigen Managementsysteme ihre Wirkung entfalten können (vgl. Aßländer 2005). Welche Maßnahmen sich bei der Korruptionsprävention in Deutschland bewährt haben, beschreiben Schneider und Ax (2006) ausführlich. Empfohlen wird eine Kombination aus personellen (z. B. Personalauswahl, Umgang mit Nebentätigkeiten, Sensibilisierung), organisatorischen (z. B. Personalrotation, Umgang mit Hinweisgebern) und strukturellen (z. B. Mehraugenprinzip, transparente Aktenführung) Maßnahmen.

Wenn Unternehmen in Staaten mit schwachen Governancestrukturen tätig sind, bewähren sich laut OECD Handbuch dieselben Maßnahmen. Der wesentliche Unterrungsorganisationen. Die Koalition wurde in Großbritannien gegründet, nachdem 1999 Global Witness einen Bericht über die Rolle der Erdölindustrie und der Banken im angolanischen Bürgerkrieges veröffentlicht hatte (vgl. Global Witness 1999). Damals stellte sich zahlreiche NGOVertreter die Frage, warum so viele rohstoffreiche Länder keine nennenswerten sozialen und ökonomischen Fortschritte erzielen. Ausgehend von der Annahme, dass eine falsche Allokation der Erträge die Ursache ist und Korruptions- sowie Betrugsdelikte hierbei eine wesentliche Rolle spielen, wurde die Forderungen nach Offenlegung aller Geldströme in diesen Industrien formuliert und die Koalition gegründet. 
schied liege dabei in dem Ausmaß an Sorgfalt und Vorsicht, das auf Seiten des Managements notwendig ist, damit präventive Maßnahmen ihre Wirkung entfalten können (vgl. OECD 2006: 12). So gilt es, die folgende Forderung aus den OECD-Leitlinien für multinationale Unternehmen in Ländern wie dem Irak mit größter Umsicht umzusetzen. Unternehmen sollen:

„1. Not offer, nor give in to demands, to pay public officials or the employees of business partners any portion of a contract payment. They should not use subcontracts, purchase orders or consulting agreements as means of channeling payments to public officials, to employees of business partners or to their relatives or business associates.

2. Ensure that remuneration of agents is appropriate and for legitimate services only. Where relevant, a list of agents employed in connection with transactions with public bodies and state-owned enterprises should be kept and made available to competent authorities“ (OECD 2000: Paragraph 6).

Im Großen und Ganzen sind es ganz einfache Maßnahmen, die Erfolg in der Korruptionsbekämpfung versprechen.

- Erstens darf man nicht von Anfang an bereit sein, Schmiergelder zu zahlen. Der Oil for Food-Skandal zeigt sehr deutlich, wie schnell ein zunächst korruptionsfreier Markt korrumpiert werden kann, wenn alle Akteure mitspielen. Die Korrumpierung eines Marktes rückgängig zu machen, ist um ein Vielfaches schwerer. Daher sollten Unternehmen in ihrem eigenen Interesse vermeiden, zur Korrumpierung beizutragen.

- Zweitens sollte im Unternehmen Klarheit herrschen, dass Schmiergeldzahlungen und Unterschlagungen nicht erwünscht sind. Führungskräfte haben hierbei eine wichtige Vorbildfunktion. Wenn der Eindruck besteht, dass Vertragsabschlüsse wichtiger sind, als die Form ihrer Anbahnung, oder dass sich jeder im Unternehmen zu seinem Vorteil bedient, sind Korruption und Betrug bereits Teil der Unternehmenskultur.

- Drittens müssen die Verantwortlichen im Unternehmen, das jeweilige Geschäftsfeld gut kennen, um sicherstellen zu können, dass Vermittler und andere Geschäftspartner des Unternehmens keine Bestechung in seinem Namen durchführen. Während Dienstleistungen - wie die Kommunikation mit Behörden und Geschäftspartnern - durch Dritte geleistet werden können, sollte das damit verbundene Know-How auch im Unternehmen vorhanden sein, damit dubiose Vorgänge als solche erkannt und verhindert werden können.

- Viertens sollten Unternehmen selbst in korrupten Märkten versuchen, neue korruptionsfreie Spielregeln einzuführen. Dies ist sicherlich keine leichte Aufgabe. Aber gerade namhafte Unternehmen verfügen über große Überzeugungskraft in Geschäftsverhandlungen und sollten daher in diesen immer wieder betonen, wie wichtig ihnen Redlichkeit und Transparenz sind und dies auch den Geschäftspartnern vorleben.

Managern sollte bei ihrer Tätigkeit immer klar sein, dass Unternehmen ihr Geschäftsumfeld beeinflussen. Dieser Einfluss kann positiv, aber auch negativ sein. Im Irak haben mehr als 2300 Unternehmen aus aller Welt dazu beigetragen, die Regierung 
Saddam Husseins zu unterstützen und damit die internationalen Sanktionen zu unterlaufen. Den Unternehmen ging es jeweils um einige wenige Vertragsabschlüsse. Aus heutiger Sicht stellt sich Frage, ob der Verlauf der Geschichte möglicherweise etwas anders ausgesehen hätte, wenn der Regierung Saddam Husseins die unterschlagenen 1,8 Milliarden US-Dollar gefehlt hätten.

\section{Literaturverzeichnis}

Aidt, T. S. (2003): Economic Analysis of Corruption: a Aurvey, in: The Economic Journal, 113(November), F632-F652.

Aßländer, M. (2005): Why the Watchdog didn’t bark. Gründe für das Moralversagen im Falle Enron, in: Forum Wirtschaftsethik, 13(2), 19-25.

Döbler, M. (2007): Anklage nur im Ausnahmefall, in: Tagesspiegel, 10. April 2007.

Eigen, P. (2002): Measuring and Combating Corruption, in: Policy Reform, 5(4), 187-201.

Gatti, C. (2007): Inquiry Links Total to Payoffs to Saddam Regime, in: International Herald Tribune, 17. Mai 2007.

Global Witness (2005): Extracting Transparency. The need for an International Financing Reporting Standard for the Extractive Industries, London: Global Witness.

Global Witness (1999): A Crude Awakening, London: Global Witness.

Homann, K./ Suchanek, A. (2005): Ökonomik. Eine Einführung, Tübingen: Mohr Siebeck.

Human Rights Watch (2000): World Report 2000. Chapter on Iraq and Iraqi Kurdistan, published under: http://www.hrw.org/wr2k1/mideast/iraq.html.

Independent Inquiry Committee into the United Nations Oil-For-Food Programme (2005a): Report on Programme Manipulation, issued on October 27, 2005, http://www.iic-offp.org/ story27oct05.htm.

Independent Inquiry Committee into the United Nations Oil-For-Food Programme (2005b): The Management of the United Nations Oil-For-Food Programme, Volume I, issued September 7, 2005, http://www.iic-offp.org/Mgmt_Report.htm.

Independent Inquiry Committee into the United Nations Oil-For-Food Programme (2005c): The Management of the United Nations Oil-For-Food Programme, Volume II, issued September 7 , 2005, http://www.iic-offp.org/Mgmt_Report.htm.

Independent Inquiry Committee into the United Nations Oil-For-Food Programme (2005d): The Management of the United Nations Oil-For-Food Programme, Volume III, issued September 7, 2005, http://www.iic-offp.org/Mgmt_Report.htm.

Independent Inquiry Committee into the United Nations Oil-For-Food Programme (2005e): The Management of the United Nations Oil-For-Food Programme, Volume IV, issued September 7, 2005, http://www.iic-offp.org/Mgmt_Report.htm.

Independent Inquiry Committee into the United Nations Oil-For-Food Programme (2005f): Report on Programme Manipulation. Explanation of Committee Tables, issued on October 27, 2005, http://www.iic-offp.org/story27oct05.htm.

Klitgaard, R. (1997): Cleaning up and Invigorating the Civil Service, in: Public Administration and Development, 17(5), 487-509.

Leyendecker, H./ Krüger, P. A. (2007): Schmiergeld, Aufgeld oder legale Provision, in: Süddeutsche Zeitung, 10. Januar 2007.

Libération (2007): Pétrole contre nourriture: une quinzaine de personnes poursuivies, in: Libération, 25. Mai 2007. 
Masters, B. (2007): US Gas Group in Bribes Deal - Oil-For-Food Programme, in: Financial Times, 8. Februar 2007.

Organisation for Economic Co-operation and Development (1998): Convention on Combating Bribery of Foreign Public Officials in International Business Transactions, DAFFE/IME/ BR(97)20, Paris: OECD.

Organisation for Economic Co-operation and Development (2000): OECD Guidelines for Multinational Enterprises, Paris: OECD.

Organisation for Economic Co-operation and Development (2006): OECD Risk Awareness Tool for Multinational Enterprises in Weak Governance Zones, Paris: OECD.

Organization of the Petroleum Exporting Countries (2006): Annual Statistical Bulletin 2005, Wien: OPEC.

Richards, A./ Waterbury, J. (1991): A Political Economy of the Middle East, Cairo: The American University in Cairo Press.

Schneider, M./ Ax, T. (Hrsg.) (2006): Rechtshandbuch Korruptionsbekämpfung, Berlin: Erich Schmidt Verlag.

Siemens (2006): Geschäftsbericht 2006, München: Siemens.

Theobald, R. (2002): Containing Corruption. Can the State Deliver? In: New Political Economy, 7(3), 435-441.

\section{Gesetzestexte und Resolutionen}

- Außenwirtschaftsgesetz der Bundesrepublik Deutschland

- Charta der Vereinten Nationen

- Code Penal der Republik Frankreich

- Resolution 661 des VN-Sicherheitsrats

- Resolution 986 des VN-Sicherheitsrat

- Strafgesetzbuch der Bundesrepublik Deutschland 